Yves Demazeau

Michal Pěchoucěk

Juan M. Corchado

Javier Bajo Pérez (Eds.)

\title{
Advances on Practical Applications of Agents and Multiagent Systems
}

9th International Conference on Practical Applications of Agents and Multiagent Systems

Springer 
\begin{tabular}{l} 
Advances in Intelligent and \\
Soft Computing \\
\hline
\end{tabular}

Editor-in-Chief: J. Kacprzyk 


\section{Advances in Intelligent and Soft Computing}

\section{Editor-in-Chief}

Prof. Janusz Kacprzyk

Systems Research Institute

Polish Academy of Sciences

ul. Newelska 6

01-447 Warsaw

Poland

E-mail: kacprzyk@ibspan.waw.pl

Further volumes of this series can be found on our homepage: springer.com

Vol. 75. X.Z. Gao, A. Gaspar-Cunha,

M. Köppen, G. Schaefer, and J. Wang (Eds.)

Soft Computing in Industrial Applications, 2010

ISBN 978-3-642-11281-2

Vol. 76. T. Bastiaens, U. Baumöl, and B.J. Krämer (Eds.)

On Collective Intelligence, 2010

ISBN 978-3-642-14480-6

Vol. 77. C. Borgelt, G. González-Rodríguez, W. Trutschnig, M.A. Lubiano, M.Á. Gil,

P. Grzegorzewski, and O. Hryniewicz (Eds.)

Combining Soft Computing and Statistical

Methods in Data Analysis, 2010

ISBN 978-3-642-14745-6

Vol. 78. B.-Y. Cao, G.-J. Wang,

S.-Z. Guo, and S.-L. Chen (Eds.)

Fuzzy Information and Engineering 2010

ISBN 978-3-642-14879-8

Vol. 79. A.P. de Leon F. de Carvalho, S. Rodríguez-González, J.F. De Paz Santana, and J.M. Corchado Rodríguez (Eds.) Distributed Computing and Artificial Intelligence, 2010

ISBN 978-3-642-14882-8

Vol. 80. N.T. Nguyen, A. Zgrzywa, and A. Czyzewski (Eds.)

Advances in Multimedia and Network

Information System Technologies, 2010

ISBN 978-3-642-14988-7

Vol. 81. J. Düh, H. Hufnagl, E. Juritsch, R. Pfliegl, H.-K. Schimany, and Hans Schönegger (Eds.)

Data and Mobility, 2010

ISBN 978-3-642-15502-4
Vol. 82. B.-Y. Cao, G.-J. Wang, S.-L. Chen, and S.-Z. Guo (Eds.) Quantitative Logic and Soft Computing 2010

ISBN 978-3-642-15659-5

Vol. 83. J. Angeles, B. Boulet, J.J. Clark, J. Kovecses, and K. Siddiqi (Eds.) Brain, Body and Machine, 2010 ISBN 978-3-642-16258-9

Vol. 84. Ryszard S. Choraś (Ed.) Image Processing and Communications Challenges 2, 2010 ISBN 978-3-642-16294-7

Vol. 85. Á. Herrero, E. Corchado, C. Redondo, and Á. Alonso (Eds.) Computational Intelligence in Security for Information Systems 2010 ISBN 978-3-642-16625-9

Vol. 86. E. Mugellini, P.S. Szczepaniak, M.C. Pettenati, and M. Sokhn (Eds.) Advances in Intelligent Web Mastering - 3, 2011 ISBN 978-3-642-18028-6

Vol. 87. E. Corchado, V. Snášel, J. Sedano, A.E. Hassanien, J.L. Calvo, and D. Ślęzak (Eds.)

Soft Computing Models in Industrial and Environmental Applications,

6th International Conference SOCO 2011

ISBN 978-3-642-19643-0

Vol. 88. Y. Demazeau, M. Pěchoucěk, J.M. Corchado, and J. Bajo (Eds.) Advances on Practical Applications of Agents and Multiagent Systems, 2011

ISBN 978-3-642-19874-8 
Yves Demazeau, Michal Pěchoucěk, Juan M. Corchado, and Javier Bajo (Eds.)

Advances on Practical Applications of Agents and Multiagent Systems

9th International Conference on Practical Applications of Agents and Multiagent Systems

算 Springer 


\section{Editors}

Prof. Yves Demazeau

Laboratoire d'Informatique de Grenoble

Centre National de la Recherche

Scientifique

Maison Jean Kuntzmann

110 av. de la Chimie

F-38041 Grenoble

France

Prof. Michal Pěchoucěk

Czech Technical University

Department of Cybernetics

Technická 2

16627 Prague 6

Czech Republic
Prof. Juan M. Corchado

Universidad de Salamanca

Departamento de Informática

y Automática

Facultad de Ciencias

Plaza de la Merced S/N

37008 Salamanca

Spain

E-mail: corchado@usal.es

Prof. Javier Bajo Pérez

Universidad Pontificia de Salamanca

Escuela Universitaria de Informática

Compañía 5

37002 Salamanca

Spain

ISBN 978-3-642-19874-8

DOI 10.1007/978-3-642-19875-5

Advances in Intelligent and Soft Computing

ISSN 1867-5662

Library of Congress Control Number: 2011923222

\section{(c)2011 Springer-Verlag Berlin Heidelberg}

This work is subject to copyright. All rights are reserved, whether the whole or part of the material is concerned, specifically the rights of translation, reprinting, reuse of illustrations, recitation, broadcasting, reproduction on microfilm or in any other way, and storage in data banks. Duplication of this publication or parts thereof is permitted only under the provisions of the German Copyright Law of September 9 , 1965 , in its current version, and permission for use must always be obtained from Springer. Violations are liable for prosecution under the German Copyright Law.

The use of general descriptive names, registered names, trademarks, etc. in this publication does not imply, even in the absence of a specific statement, that such names are exempt from the relevant protective laws and regulations and therefore free for general use.

Typeset \& Cover Design: Scientific Publishing Services Pvt. Ltd., Chennai, India.

Printed on acid-free paper

543210

springer.com 


\section{Preface}

Research on Agents and Multi-Agent Systems has matured during the last decade and many effective applications of this technology are now deployed. An international forum to present and discuss the latest scientific developments and their effective applications, to assess the impact of the approach, and to facilitate technology transfer, has become a necessity.

PAAMS, the International Conference on Practical Applications of Agents and Multi-Agent Systems is the international yearly tribune to present, to discuss, and to disseminate the latest developments and the most important outcomes related to real-world applications. It provides a unique opportunity to bring multidisciplinary experts, academics and practitioners together to exchange their experience in the development of Agents and Multi-Agent Systems.

This volume presents the papers that have been accepted for the 2011 edition. These articles capture the most innovative results and this year's trends: Finance and Trading, Information Systems and Organisations, Leisure Culture and Interactions, Medicine and Cloud Computing, Platforms and Adaptation, Robotics and Manufacturing, Security and Privacy, Transports and Optimisation paper has been reviewed by three different reviewers, from an international committee composed of 75 members from 24 different countries. From the 81 submissions received, 15 were selected for full presentation at the conference, and 24 were accepted for short presentation at the conference.

We would like to thank all the contributing authors, as well as the members of the Program Committee and the Organizing Committee for their hard and highly valuable work. Their work has helped to contribute to the success of the PAAMS'11 event. Thanks for your help, PAAMS'11 wouldn't exist without your contribution.

Yves Demazeau

Frank Dignum

PAAMS'11 Program Co-chairs
Juan Manuel Corchado Javier Bajo

PAAMS'11 Organizing Co-chairs 



\section{Organization}

\section{General Co-chairs}

Yves Demazeau

Michal Pěchouček (Co-chair)

Juan M. Corchado

Javier Bajo

\section{Advisory Board}

Juan Pavón

Frank Dignum

\section{Program Committee}

Yves Demazeau (Co-chair)

Michal Pěchouček (Co-chair)

Carole Adam

Frederic Amblard

Francesco Amigoni

Luis Antunes

Javier Bajo

Zbigniew Banaszak

Fabio Luigi Bellifemine

Olivier Boissier

Juan A. Botía

Vicente Botti

Stefano Bromuri

Sven Brueckner

Valerie Camps

Longbing Cao
Centre National de la Recherche Scientifique (France)

Czech Technical University in Prague (Czech Republic)

University of Salamanca (Spain)

Pontifical University of Salamanca (Spain)

Universidad Complutense de Madrid (Spain)

Utrecht University (The Netherlands)

Centre National de la Recherche Scientifique (France)

Czech Technical University in Prague

(Czech Republic)

University of Grenoble (France)

University of Toulouse (France)

Politecnico di Milano (Italy)

University of Lisbon (Portugal)

Pontifical University of Salamanca (Spain)

Warsaw University of Technology (Poland)

Telecom Italia (Italy)

Ecole Nationale Superieure des Mines de Saint Etienne (France)

University of Murcia (Spain)

Polytechnic University of Valencia (Spain)

University of Applied Sciences Western

Switzerland (Switzerland)

Jacobs Technology Inc. (USA)

University of Toulouse (France)

University of Technology Sydney (Australia) 
Lawrence Cavedon

Pierre Chevaillier

Juan Manuel Corchado

Keith Decker

Frank Dignum

Virginia Dignum

Alexis Drogoul

Julie Dugdale

Edmund Durfee

Partha S Dutta

Amal Elfallah

Torsten Eymann

Rubén Fuentes

Francisco Garijo

Khaled Ghedira

Sylvain Giroux

Pierre Glize

Vladimir Gorodetski

Dominic Greenwood

Kasper Hallenborg

Benjamin Hirsch

Tom Holvoet

Shinichi Honiden

Jomi Hübner

Toru Ishida

Takayuki Ito

Vicente Julian

Achilles Kameas

Jeffrey O. Kephart

Franziska Kluegl

Matthias Klusch

Martin Kollingbaum

Jaroslaw Kozlak

Zakaria Maamar

Rene Mandiau

Philippe Mathieu

Eric Matson

Fabien Michel

José M. Molina

Mirko Morandini

Joerg Mueller
RMIT Melbourne (Australia)

University of Brest (France)

University of Salamanca (Spain)

University of Delaware (USA)

Utrecht University (The Netherlands)

Utrecht University (The Netherlands)

Institut de Recherche pour 1 Developpement (Vietnam)

University of Grenoble (France)

University of Michigan (USA)

Rolls-Royce (United Kingdom)

University of Paris 6 (France)

University of Bayreuth (Germany)

University Computense de Madrid (Spain)

Telefonica I+D (Spain)

National School of Computer Sciences (Tunisia)

Unversity of Sherbrooke (Canada)

University of Toulouse (France)

University of Saint Petersburg (Russia)

Whitestein Technologies (Switzerland)

University of Southern Denmark (Denmark)

Technical University Berlin (Germany)

Catholic University of Leuven (Belgium)

National Institute of Informatics Tokyo (Japan)

Federal University of Santa Catarina (Brazil)

University of Kyoto (Japan)

Massachussets Institute of Technology (USA)

Polytechnic University of Valencia (Spain)

University of Patras (Greece)

IBM Research (USA)

University of Örebro (Sweden)

DFKI (Germany)

University of Aberdeen (United Kingdom)

University of Science and Technology in Krakow (Poland)

Zayed University (United Arab Emirates)

University of Valenciennes (France)

University of Lille (France)

Purdue University (USA)

University of Reims (France)

Universidad Carlos III de Madrid (Spain)

University of Trento (Italy)

Clausthal University of Technology (Germany) 
Peter Novak

Eugenio Oliveira

Sascha Ossowski

Julian Padget

Adolfo López Paredes

Juan Pavón

Jeremy Pitt

Juan Rodriguez Aguilar

Simon Thompson

Paolo Torroni

Rainer Unland

Domenico Ursino

Birna van Riemsdijk

Jacques Verriet

Jiri Vokrinek

Danny Weyns

Niek Wijngaards

Gaku Yamamoto

\section{Organizing Committee}

Juan M. Corchado (Chairman) Javier Bajo (Co-Chairman)

Juan F. De Paz

Sara Rodríguez

Dante I. Tapia

Emilio Corchado

Fernando de la Prieta Pintado

Davinia Carolina Zato

Domínguez
Czech Technical University in Prague (Czech Republic)

University of Porto (Portugal)

University of Rey Juan Carlos (Spain)

University of Bath (United Kingdom)

University of Valladolid (Spain)

Universidad Complutense de Madrid (Spain)

Imperial College of London

(United Kingdom)

Artificial Intelligence Research Institute (Spain)

British Telecom IIS Research Centre

(United Kingdom)

University of Bologna (Italy)

University of Duisburg (Germany)

University of Reggio Calabria (Italy)

University of Delft (The Netherlands)

Embedded Systems Institute

(The Netherlands)

Czech Technical University in Prague

(Czech Republic)

Catholic University of Leuven (Belgium)

Thales, D-CIS lab (The Netherlands)

IBM and Tokyo Institute of Tecnology

(Japan)

University of Salamanca (Spain)

Pontifical University of Salamanca (Spain)

University of Salamanca (Spain)

University of Salamanca (Spain)

University of Salamanca (Spain)

University of Salamanca (Spain)

University of Salamanca (Spain)

University of Salamanca (Spain) 



\section{Contents}

\section{Information and Organisations}

A J-MADeM Agent-Based Social Simulation to Model Urban Mobility

Francisco Grimaldo, Miguel Lozano, Fernando Barber,

Alejandro Guerra-Hernández

Diversity of the Knowledge Base in Organizations: Results of an Agent-Based Simulation

Friederike Wall

Weighing Communication Overhead against Travel Time

Reduction in Advanced Traffic Information Systems .........

Rutger Claes, Tom Holvoet

Information Extraction System Using Indoor Location and Activity Plan .................................. 33
Bjørn Grønbck, Pedro Valente, Kasper Hallenborg

Using Multi-Agent Systems to Visualize Text

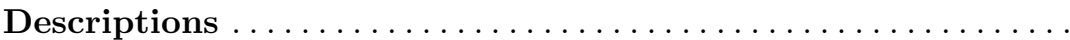

Edgar Bolaño-Rodríguez, Juan C. González-Moreno,

David Ramos-Valcarcel, Luiz Vázquez-López

\section{Robotics and Manufacturing}

Empowering Adaptive Manufacturing with Interactive Diagnostics: A Multi-Agent Approach

Thomas M. Hubauer, Christoph Legat, Christian Seitz 
Multi-Agent Cooperation for Advanced Teleoperation of an Industrial Forklift in Real-Time Environment........... 57 F.J. Serrano Rodríguez, J.F. Rodríguez-Aragón, B. Curto Diego, V. Moreno Rodilla

Map Partitioning to Approximate an Exploration Strategy in Mobile Robotics ................................. 63 Guillaume Lozenguez, Lounis Adouane, Aurélie Beynier, Philippe Martinet, Abdel-Illah Mouaddib

ROAR: Resource Oriented Agent Architecture for the Autonomy of Robots ........................... 73 Arnaud Degroote, Simon Lacroix

Engineering Agent Frameworks:An Application in Multi-Robot Systems

Jérôme Lacouture, Victor Noël, Jean-Paul Arcangeli,

Marie-Pierre Gleizes

\section{Security and Privacy}

Lightweight Trusted Routing for Wireless Sensor

Networks

Laurent Vercouter, Jean-Paul Jamont

Privacy-Preserving Strategy for Negotiating Stable, Equitable and Optimal Matchings

Maxime Morge, Gauthier Picard

Searching Flocks in Peer-to-Peer Networks

Hugo Pommier, Benoît Romito, François Bourdon

Securing Mobile Agents via Combining Encrypted

Functions

Hamed Aouadi

A Multi-Agent System with Distributed Bayesian Reasoning for Network Fault Diagnosis

Álvaro Carrera, Javier Gonzalez-Ordás, Javier García-Algarra,

Pablo Arozarena, Mercedes Garijo

\section{Platforms and Adaptation}

Using TraSMAPI for Developing Multi-Agent Intelligent Traffic Management Solutions Ivo J.P.M. Timóteo, Miguel R. Araújo, Rosaldo J.F. Rossetti,

Eugénio C. Oliveira 
EPIS: A Grid Platform to Ease and Optimize Multi-agent Simulators Running ............................... 129 E. Blanchart, C. Cambier, C. Canape, B. Gaudou, T.-N. Ho, T.-V. Ho, C. Lang, F. Michel, N. Marilleau, L. Philippe

Formal Specification of Holonic Multi-Agent Systems: Application to Distributed Maintenance Company ...

Belhassen Mazigh, Vincent Hilaire, Abderrafiaa Koukam

Controlling Bioprocesses Using Cooperative Self-organizing

Agents ...................................... 141

Sylvain Videau, Carole Bernon, Pierre Glize, Jean-Louis Uribelarrea

Towards Context-Based Inquiry Dialogues for Personalized Interaction

Helena Lindgren

\section{Transports and Optimisation}

Modelling Driver Interdependent Behaviour in

Agent-Based Traffic Simulations for Disaster Management ... 163 David Handford, Alex Rogers

Agent-Based Integrated Decision Making for Autonomous Vehicles in Urban Traffic ......................... 173 Maksims Fiosins, Jelena Fiosina, Jörg P. Müller, Jana Görmer

Towards Urban Traffic Regulation Using a Multi-Agent System Neila Bhouri, Flavien Balbo, Suzanne Pinson

Application of Holonic Approach for Transportation Modelling and Optimising ......................... 189 Jarostaw Koźlak, Sebastian Pisarski, Matgorzata Żabinska

A Multiagent System Approach to Grocery Shopping. 195 Hongying Du, Michael N. Huhns

\section{Leisure, Culture, Interactions}

Artificial Snow Optimization in Winter Sport Destinations Using a Multi-agent Simulation ...................... 201 M. Revilloud, J.-C. Loubier, M. Doctor, M. Kanevski, V. Timonin, M. Schumacher 
Middle Age Social Networks: A Dynamic Organizational Study.......................................... 211

L. Lacomme, V. Camps, Y. Demazeau, F. Hautefeuille, B. Jouve

From an Individual Perspective to a Team Perspective in Agent-Based Negotiation .......................... 217

Víctor Sánchez-Anguix, Vicente Julián, Ana García-Fornes

An Exploratory Analysis for Designing Robust Interactions in Multi-Agent Systems ............................. 225

Celia Gutiérrez and Iván García-Magariño

Comparison of DCSP Algorithms: A Case Study for

Multi-agent Exploration ......................... 231

Pierre Monier, Arnaud Doniec, Sylvain Piechowiak, René Mandiau

\section{Medicine and Cloud Computing}

Heterogeneous Multiagent Architecture for Dynamic Triage of Victims in Emergency Scenarios .............. 237 Estanislao Mercadal, Sergi Robles, Ramon Martí, Cormac J. Sreenan, Joan Borrell

A Decision Support System for Hospital Emergency Departments Built Using Agent-Based Techniques . Manel Taboada, Eduardo Cabrera, Emilio Luque

An Agent-Based Dialog Simulation Technique to Develop and Evaluate Conversational Agents

David Griol, Nayat Sánchez-Pi, Javier Carbó, José M. Molina

Cloud Computing Service for Managing Large Medical

Image Data-Sets Using Balanced Collaborative Agents ...... 265

Raúl Alonso-Calvo, Jose Crespo, Victor Maojo, Alberto Muñoz,

Miguel García-Remesal, David Pérez-Rey

Software Agents as Cloud Computing Services .

Ignacio Lopez-Rodriguez and Mario Hernandez-Tejera

\section{Finance and Trading}

Efficient Monitoring of Financial Orders with Agent-Based

Technologies

Philippe Mathieu and Olivier Brandouy

An Agent Task Force for Stock Trading. 
Contracts Negotiation in Market Environments Taking into Consideration the Strength of Each Economic Player . . . . . . 299 Eukasz Jankowski, Jarostaw Koźlak, Matgorzata Żabińska

Bid Definition Method for Electricity Markets Based on an Adaptive Multiagent System

Tiago Pinto, Zita Vale, Fátima Rodrigues, Hugo Morais, Isabel Praça

Author Index . 

The series "Advances in Intelligent and Soft Computing" contains publications on various areas within the so-called soft computing, which include fuzzy sets, rough sets, neural networks, evolutionary computations, probabilistic and evidential reasoning, multi-valued logic, and related fields. The publications within "Advances in Intelligent and Soft Computing" are primarily textbooks and proceedings of important conferences, symposia and congresses. They cover significant recent developments in the field, both theoretical and applied. An important feature of the series is the short publication time and world-wide distribution, allowing a rapid and broad dissemination of research results.

Yves Demazeau · Michal Pěchoucěk · Juan M. Corchado Javier Bajo Pérez (Eds.) Advances on Practical Applications of Agents and Multiagent Systems

PAAMS, the International Conference on Practical Applications of Agents and Multi-Agent Systems is the international yearly tribune to present, to discuss, and to disseminate the latest developments and the most important outcomes related to real-world applications. It provides a unique opportunity to bring multi-disciplinary experts, academics and practitioners together to exchange their experience in the development of Agents and Multi-Agent Systems. This volume presents the papers that have been accepted for the 2011 edition. These articles capture the most innovative results and this year's trends: Finance and Trading, Information Systems and Organisations, Leisure Culture and Interactions, Medicine and Cloud Computing, Platforms and Adaptation, Robotics and Manufacturing, Security and Privacy, Transports and Optimisation paper.

ISSN $1867-5662$

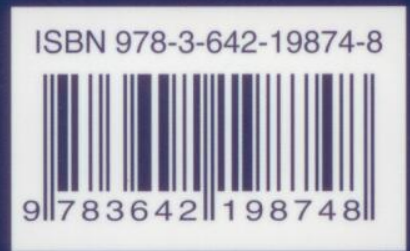

\section{Available online} springerlink.com

springer.com 